\title{
Radio continuum spectra of gigahertz-peaked spectrum galaxies ${ }^{\star} \star \star$
}

\author{
I. Torniainen ${ }^{1}$, M. Tornikoski ${ }^{1}$, A. Lähteenmäki ${ }^{1}$, M. F. Aller ${ }^{2}$, H. D. Aller ${ }^{2}$, and M. G. Mingaliev ${ }^{3}$ \\ 1 Metsähovi Radio Observatory, Helsinki University of Technology, Metsähovintie 114, 02540 Kylmälä, Finland \\ e-mail: ilo@kurp.hut.fi \\ 2 Department of Astronomy, University of Michigan, Ann Arbor, MI, 48109, USA \\ 3 Special Astrophysical Observatory, Russian Academy of Sciences, Nizhnij Arkhyz, Karachaevo-Cherkesia 369167, Russia
}

Received 7 December 2006 / Accepted 13 March 2007

\section{ABSTRACT}

\begin{abstract}
Context. Recent studies have shown that a remarkable share of quasars classified in the literature as gigahertz-peaked spectrum (GPS) sources and high frequency peakers (HFPs) are actually flaring flat-spectrum sources or blazars. Thus, at least among the quasar-type samples, genuine GPS sources and HFPs seem to be rare.

Aims. We have studied variability and the shape of the radio continuum spectra of a sample of 96 galaxy-type GPS sources and HFPs in order to find out whether there is a similar contamination in the galaxy-type samples.

Methods. We collected radio data for the sample from the literature, our long-term monitoring campaigns, and recent observations, and then plotted the radio continuum spectra. We also calculated the peak frequencies, the spectral indices, and the variability indices, and finally classified the sources according to these parameters.

Results. About $30 \%$ of the galaxies in our sample are clearly GPS sources, for another $\sim 30 \%$ there are not enough data for a solid classification, and the rest are flat- or steep-spectrum sources.

Conclusions. The galaxy-type GPS samples seem to be cleaner than the quasar-type, but there is also a remarkable contamination of other source types among the galaxies. However, there may be a strong selection effect, originating from the different selection criteria of the original samples, which must be taken into consideration when comparing the results of this and our previous study. Both simultaneous spectra and long-term monitoring are essential when classifying convex-spectrum sources. However, even monitoring for several years may not reveal the variable nature of a source with a convex radio spectrum.
\end{abstract}

Key words. quasars: general - radio continuum: galaxies

\section{Introduction}

The gigahertz-peaked spectrum (GPS) sources are small and luminous active galactic nuclei with the distinctive feature of a convex shape of the radio continuum spectrum, peaking at GHz-frequencies. The GPS sources are associated with either quasars or galaxies. At least some of them are related to compact steep-spectrum (CSS) sources, which are larger and have a lower peak frequency, and to high frequency peakers (HFP), which are smaller and have a higher peak frequency. For a review of GPS and CSS sources, see O'Dea (1998). Throughout this paper, we follow the notation of O'Dea (1998) and consider the turnover frequency of $500 \mathrm{MHz}$ (in the rest frame) to be the borderline between GPS and CSS sources.

The currently leading theory explaining the nature of GPS and CSS sources and HFPs is the so-called youth scenario (e.g., Phillips \& Mutel 1982; Fanti et al. 1995; Murgia et al. 1999; Fanti et al. 2000; Dallacasa et al. 2000). It argues that the HFPs are newborn radio sources that will evolve into extended radio sources via GPS and CSS stages. It is possible that the activity is recurrent in at least some sources: there have been observations

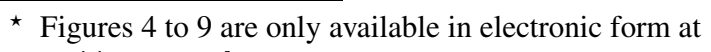
http: //www . aanda.org

$\star \star$ Table 2 is only available in electronic form at the CDS via anonymous ftp to cdsarc.u-strasbg.fr $(130.79 .128 .5)$ or via http://cdsweb.u-strasbg.fr/cgi-bin/qcat? J/A+A/469/451
}

of faint extended emission around a few GPS sources (e.g. Baum et al. 1990; Stanghellini et al. 1990; Marecki et al. 2003). The extended emission could be a relic of an earlier active period, in which the reborn radio jets are expanding.

Another explanation for the compact size is the frustration scenario, in which the dense ambient material prevents the radio jets from propagating outside the host galaxy. There have been observations and calculations both for and against this scenario. Gopal-Krishna \& Wiita (1991) suggested that CSS sources would have acquired remarkable amounts of gas by galaxy interactions. Some studies support this result (e.g., Mantovani et al. 1994), but several disagree with it (e.g., Fanti et al. 1995; O’Dea et al. 1996, 2005).

The GPS galaxies and GPS quasars are considered to be physically different sources, only having a similar spectral shape in the radio continuum. This has been argued earlier by, among other, Snellen et al. (1999) and Stanghellini et al. (2001). Recent results by Stanghellini et al. (2005) and Orienti et al. (2006) support this view. Stanghellini et al. (2005) studied extended emission around GPS sources and conclude that, if a GPS quasar exhibits a core-jet or a complex VLBI morphology, it is likely that it represents an intermediately beamed full-size radio source related to common flat-spectrum radio sources. Thus, it would not be related to truly compact and young/recurrent/frustrated GPS galaxies at all. Orienti et al. (2006) studied VLBI images of quasar and galaxy type HFPs, and they confirm that the 
convex shape of the spectrum originates from mini-lobes and/or hot-spots in galaxy-type sources and from the inner regions near the core and from the origins of the jets in quasar-type sources.

Bolton et al. (2006) studied the variability of a sample of 9C sources at $1.4 \mathrm{GHz}, 4.8 \mathrm{GHz}$, and $15 \mathrm{GHz}$, including HFPs and GPS sources. The sample included both galaxies and quasars. They found that at least 13 out of 18 HFPs were variable at some of the frequencies. None of the GPS sources were variable at 15 $\mathrm{GHz}$ during the 3-year study, but 10 out of 24 showed evidence of variability at lower frequencies. They also found the percentage of the GPS sources and HFPs to be too high to be explained solely with the evolutionary youth scenario. They suggested that a fair amount of the GPS sources and HFPs are not intrinsically young sources but dominated by beamed, self-absorbed components.

It is interesting to note, however, that the aforementioned apparent "over-abundance" of very compact sources does not vanish even if their morphology is taken into account so that the effects introduced by the projection are suppressed. Such a study was carried out by Gugliucci et al. (2005), who measured the kinematic ages of compact symmetric objects (CSO), a morphological class that many GPS galaxies are associated with, and found that the fraction of very young $(<500 \mathrm{yr})$ CSOs was too large if a uniform age distribution of a steady-state population was assumed. Hence, they concluded that it is possible that the CSOs undergo phases of activity of only several hundred years. They also find that three of their CSOs exhibited unexpectedly high variability (30\% in five years).

Earlier, we studied long-term variability and the shape for the spectrum of two samples of mostly quasar-type GPS sources, and also tried to identify new high-peaking convex-spectrum sources. In Tornikoski et al. (2001) we presented radio spectra of 12 quasar-type GPS candidates, 14 GPS sources identified in the literature and eight other sources with inverted spectra. This sample was constructed from the literature and our high-frequency monitoring data observed with the Swedish-ESO Submillimetre Telescope. The variability of most of the sources, both GPS sources and candidates, was found to be large, and it was evident that the variability of flat-spectrum sources may lead to misidentifications based on spectra constructed from data obtained during a transient gigahertz-peaked spectral phase.

In Torniainen et al. (2005, hereafter Paper I), we studied a sample of northern GPS sources and candidates, mostly quasars, selected from the Metsähovi monitoring project. We found out that only two quasars and one empty field object out of 29 previously identified, quasar-type GPS or HFP sources maintained their GPS or HFP classification, whereas a remarkable fraction of the GPS and HFP quasars identified in the literature were not genuine convex spectrum sources at all, but rather flaring flatspectrum sources that were classified as GPS sources or HFPs based on inadequate data. This result is in accordance with the result of Tinti et al. (2005), who noticed that some of the HFP sources were actually flaring blazars caught in a flaring state. Thus, for a reliable classification, it is necessary to have both simultaneous multifrequency data in the radio band to define the turnover, preferably at several epochs, and long-term monitoring at several frequencies to determine whether there is variability at any or all of the frequencies. The conclusions drawn from studies of contaminated quasar-type samples must be taken with caution.

In this paper we adopt the same approach as in Paper I but for galaxy-type GPS sources. Our aim is to study the variability behaviour and the shape of the spectrum of the GPS galaxies in order to discover whether there is any contamination from variable flat-spectrum sources or other source populations among them.

\section{Sample and observations}

We have gathered a sample of 96 galaxy-type GPS or HFP sources (for simplicity, hereafter also referred to as GPS sources) from the literature. We included all the main GPS samples, but left out the faint sample of Snellen et al. (1998), which was expected to be too faint to be observed with the Metsähovi telescope, where the detection limit is $0.2 \mathrm{Jy}$ at $37 \mathrm{GHz}$ under optimal conditions.

The sources and their characteristics are presented in Table 2, available at the CDS. It contains the following information. Column 1 lists the name of the source, Col. 2 the redshift, Cols. 3 and 4 give the peak frequency of the spectrum in the observers frame and in the rest frame, respectively. Column 5 lists the greatest fractional variability and Col. 6 gives the number of observations and Col. 7 the center frequency of the data bin with the greatest variability index. Columns 8 and 9 list the spectral index below and above the peak frequency, respectively, and Col. 10 gives the classification proposed in this paper.

Some of the sources have been observed in the framework of the University of Michigan variability programme since the 1970s and as a part of the Metsähovi monitoring project since the mid-1980s, but some sources were observed only a few times at Metsähovi in the beginning of 2006. There is a small overlap with the sample of Paper I: there were three galaxies: B0238-084, B0742+103, and B2021+614, in the Paper I "bona fide" sample, since the original selection was not made based on the optical identification. These three sources have also been chosen for this study but are not taken into account in the statistics of Paper I when comparing the results of these two papers in Sect. 5.

The observations reported in this paper were made with the 14-m Metsähovi radio telescope at a frequency of $37 \mathrm{GHz}$. The observations and the data reduction are described in Teräsranta et al. (1998).

Some of the sources were also observed in the University of Michigan Radio Astronomy Observatory. The University of Michigan variability programme uses a $26-\mathrm{m}$ prime-focus paraboloid equipped with radiometers operating at centre frequencies of 4.8, 8.0, and $14.5 \mathrm{GHz}$. Details of the calibration and analysis techniques are described in Aller et al. (1985).

In addition, data were collected from the literature by using the CATS database (Verkhodanov et al. 1997). For the spectral fits, some high flux density data points at low frequencies were removed since they are not considered part of the convex shape of the spectrum.

\section{Analysis}

Since there are sources with abundant monitoring data at some frequencies and very sparsely sampled data at others, straightforward fitting of the spectrum would have produced results biased towards the monitoring frequencies. Also, studying variability at each single frequency band at a time would have produced reliable estimates of the source variability only if there were sufficiently sampled data for all the sources at all frequency bands, which is not the case.

To overcome these drawbacks, we created a simple script that bins the data at similar frequencies together and calculates the parameters for each bin. The frequency axis was divided into 
logarithmically equidistant intervals, chosen to be as wide as the fractional interval between $7 \mathrm{GHz}$ and $10 \mathrm{GHz}$. The data at each interval was bound to the logarithmic centre of the interval, and, for example, the median of the flux density and the fractional variability index $\left(\operatorname{Var}_{\Delta S}=\left(S_{\max }-S_{\min }\right) / S_{\min }\right)$ were calculated. This way the data scattered at slightly differing frequencies will all contribute to the parameters; for example, the variability index and the biasing effect of having different numbers of observations at each frequency is decreased.

For the calculation of the parameters, as well as for the plotting, we excluded poorly documented data points that were abnormally high or low. The uncertainties of the measurements, typically of the order of $0.1 \mathrm{Jy}$, are not included in the calculation of the variability index since the error estimates were not available for all the data in the CATS database and also for compatibility with Paper I where the same method was used.

To model the shape of the spectra, we fitted the following equation from Kovalev et al. (2000), rearranged by Dallacasa et al. (2000), to the median flux density of each data bin,

$\log S=a-\sqrt{\left.b^{2}+(c \log v-d)^{2}\right)}$.

In general, Eq. (1) models the spectra in this sample very well, but since it does not model any physical properties, we made some adjustments to the fits of some sources, as described below.

Some of the sources in our sample have abundant data on both sides of the turnover, so that the flux density peak and the turnover frequency are easy to determine and there are no difficulties in interpreting the fits. However, there are a number of sources in which the fits from Eq. (1) do not represent the slope of the data accurately, and for those sources picked up by visual examination, logarithmic linear fits were also applied. In some of these cases the flat top of the peak was not used for the linear fit in order to model the declining slope better.

Admittedly, the majority of the sources have insufficient data for determining both spectral indices reliably, or even at all. For some sources the optically thin part of the spectrum is available and the spectral index is calculated either with Eq. (1) or linearly. Some sources only show a very wide round top of the convex shape, and it is not reasonable to fit any spectral indices to this kind of data. Using Eq. (1), the slopes are calculated from extrapolated values as follows. When applicable, frequencies $0.1 \mathrm{MHz}$ and $1 \mathrm{MHz}$ are selected to represent the optically thick and $100 \mathrm{GHz}$ and $1000 \mathrm{GHz}$ the optically thin part of the spectrum. These values are substituted into Eq. (1) to get the respective, modelled flux density values, and the basic formula for the slope in the logarithmic scales is used to derive the spectral indices: $\alpha=\left(\log S_{2}-\log S_{1}\right) /\left(\log v_{2}-\log v_{1}\right)$. In Figs. $2-9^{1}$ the radio data and the fits are shown for all the sources. If a linear fit is plotted, then the related spectral index ( $\alpha_{\text {below }}$ below and $\alpha_{\text {above }}$ above the turnover) is determined from the linear fit, otherwise Eq. (1) is used. The parameters derived from the fits are presented in Table 2.

The sources were classified using the following criteria. Sources with the spectral index below the turnover $\alpha_{\text {below }}>0.5$ and $\operatorname{Var}_{\Delta S}<3$ were classified as true GPS sources (label gps in Tables 1 and 2). For GPS classification, we also required that there were sufficient data on both sides of the turnover, so that the curve fitting and thus the determination of the spectral indices were not relying on a single data point. Although there seems to be only one data point in the optically thick part of the

1 Figures 4-9 are only available in the electronical edition of the journal. spectra of two sources, B0022-423 and B0439-337, there are in fact two very similar points originating from different references. For that reason we decided to classify them as GPS sources.

Sources were labelled with $n$ meaning "not enough data" when they had spectra showing a convex shape and a fitted curve peaking near $\mathrm{GHz}$-frequencies but belonging to the aforementioned case of too sparse data for any solid conclusions. This means that these sources could possibly be classified as GPS sources with more complete datasets. Also, the variability limit $\operatorname{Var}_{\Delta S}<3$ was required for this class, more variable sources were classified according to the spectral index. Sources with a convex shape but with $\alpha_{\text {below }}<0.5$ or $\operatorname{Var}_{\Delta S}>3$ - or both - were classified as convex ( $c$ or $c, v$, respectively, in Tables 1 and 2).

Sources with spectral indices in the range $-0.5-+0.5$ were labelled as flat spectrum sources $(f)$ and sources with $\alpha<-0.5$ as steep spectrum sources $(s)$. There were 16 sources with a flat plateau at low frequencies and a steep fall at high frequencies, and they were marked with $f / s$. For these sources there is a possibility that at very low frequencies there is a steep rise in the spectrum; but even in such case, these sources would not be GPS sources but rather CSS sources due to the low frequency of the turnover.

\section{Results}

Using the above-mentioned criteria, 29 positive GPS identifications were found among the 96 earlier classified galaxy-type GPS sources, which are $30.2 \%$ of the total sample. Additionally, there were 28 possible identifications that could not be confirmed due to inadequate data sets, corresponding to $29.2 \%$. The spectra of the remaining sources were either too variable or their spectra were

$$
\begin{aligned}
& \text { - too flat, or } \\
& \text { - did not have a clear inverted part, or } \\
& \text { - the frequencies of their possible turnovers were too low for } \\
& \text { a gigahertz-peaked label to be justified. }
\end{aligned}
$$

The parameters for each class can be found in Table 1. The variability index $\operatorname{Var}_{\triangle S \text {,MAX }}$ presented in Table 1 is the average of the greatest values of each source, regardless of the frequency of the bin. The last column presents the mode of the frequency of the bin with the highest variability index. Since it is the central frequency of the bin, it does not necessarily match any of the actual observing frequencies precisely.

\subsection{Notes on some individual sources}

For the source B0019-000 (Fig. 2), the dataset extends only to few hundred megahertz below the turnover at $0.76 \mathrm{GHz}$ and the index of the optically thick part of the spectrum $\alpha_{\text {below }}=0.46$ is slightly below the GPS limit. With suitable low-frequency data, the source could turn out to be a GPS source meeting our criteria, but for the time being we labelled the spectrum of this source as "convex".

The source B0026+346 is listed as a flat-spectrum object with an apparent jet speed of $4.7 c$ in the MOJAVE programme ${ }^{2}$ archive. The spectrum is said to be too flat at high frequencies for a GPS source. However, with the inclusion of our data in Fig. 2 the slopes are steep enough and the variability low enough to meet our criteria, and the source is classified as a GPS source,

\footnotetext{
${ }^{2}$ http://Www.physics.purdue.edu/astro/MOJAVE/ index.html
} 
Table 1. Average parameters for different classes of sources proposed in this paper. For the classification criteria, see Sect. 3.

\begin{tabular}{l|rrrrrrrr}
\hline \hline $\begin{array}{l}\text { Proposed } \\
\text { class }\end{array}$ & $N$ & $z$ & $v_{\text {peak,OBS }}$ & $v_{\text {peak,REST }}{ }^{1}$ & $\alpha_{\text {below }}$ & $\begin{array}{r}\alpha_{\text {above }} \\
\text { GHax }\end{array}$ & $\begin{array}{r}\text { Var }_{\Delta S} \\
\text { GHz }\end{array}$ & Mode of $v_{\operatorname{Var}_{\Delta S, M A X}}$ \\
\hline gps & 29 & 0.59 & 2.77 & 3.86 & +0.91 & -0.88 & 1.21 & 36.7 \\
$\mathrm{n}$ & 28 & 0.40 & 1.53 & 2.82 & +0.78 & -0.91 & 0.52 & 0.4 \\
$\mathrm{~s}$ & 11 & 0.37 & 0.36 & 0.50 & - & -0.84 & 0.78 & 4.3 \\
$\mathrm{f}$ & 7 & 1.07 & 2.01 & 4.54 & +0.36 & -0.34 & 1.50 & 4.3 \\
$\mathrm{f} / \mathrm{s}$ & 16 & 0.78 & 0.35 & 0.61 & +0.29 & -0.90 & 0.86 & 4.3 \\
$\mathrm{c}$ & 5 & 0.42 & 2.16 & 3.10 & +1.15 & -0.95 & 3.34 & 36.7 \\
all & 96 & 0.58 & 1.65 & 2.77 & +0.82 & -0.86 & 1.04 & 4.3 \\
\hline
\end{tabular}

${ }^{1}$ Calculated when $z$ available; gps $=$ Gigahertz-peaked spectrum; $\mathrm{n}=$ Not enough data for GPS identification; $\mathrm{s}=$ Steep spectrum; $\mathrm{f}=$ Flat spectrum; $\mathrm{f} / \mathrm{s}=$ Flat at low frequencies, steep at high frequencies; $\mathrm{c}=$ Convex (and variable) spectrum.
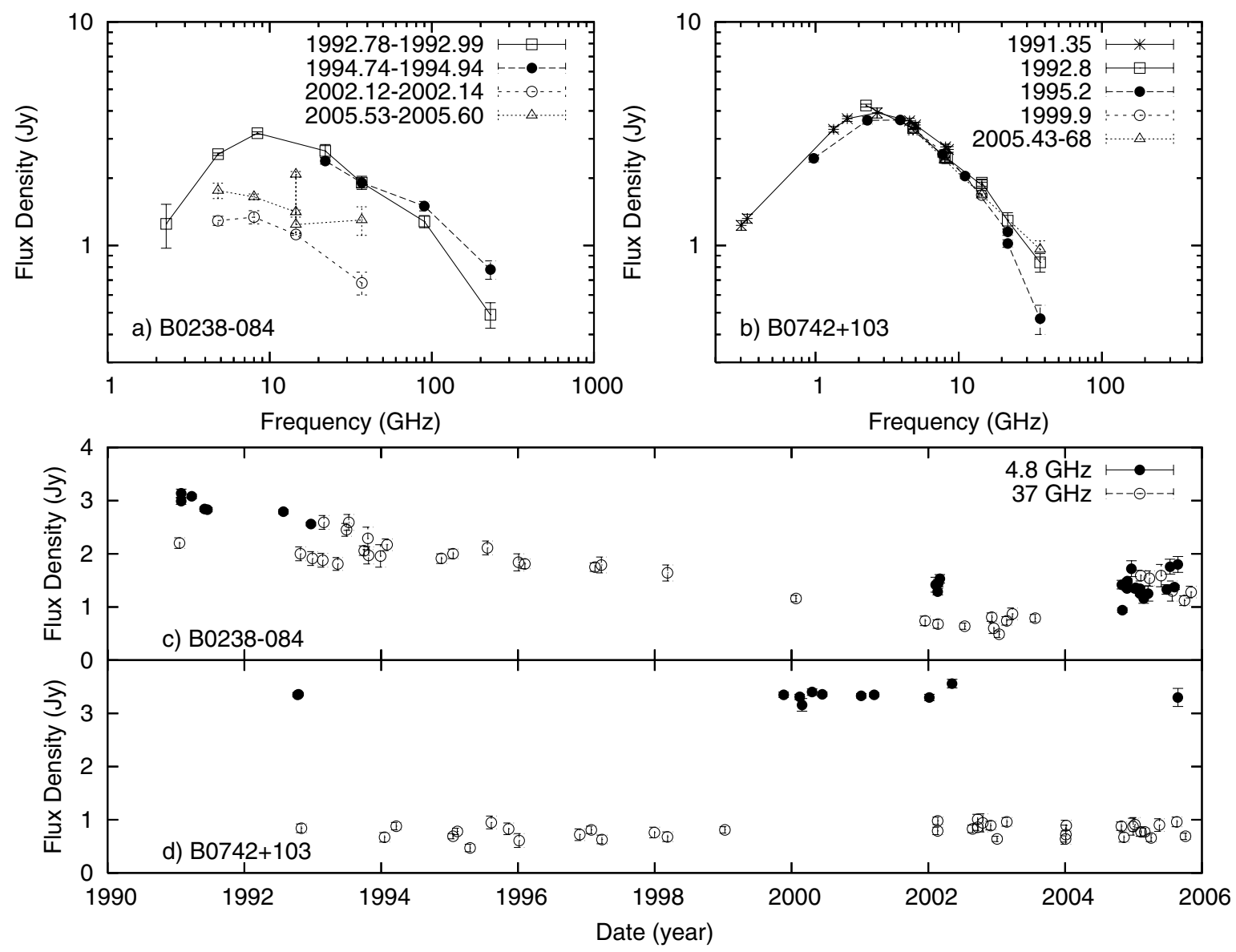

Fig. 1. Semisimultaneous spectra of a) B0238-084 and b) B0742+103. The bottom panels c) and d) show the light curves for the sources at 4.8 $\mathrm{GHz}$ and $37 \mathrm{GHz}$.

even though high apparent speeds are not considered to be typical of GPS sources.

B0238-084, also known as NGC 1052, is an elliptical galaxy and the nearest object in the sample $(z=0.005)$. It was referred to as a GPS source in de Vries et al. (1997). It has the most prominent variability in our sample: a factor of 3 at several frequencies with $\operatorname{Var}_{\triangle S, \operatorname{MAX}}=4.57$ for the data bin centred at $8.8 \mathrm{GHz}$. The overall shape of the spectrum (Fig. 2) is clearly not gigahertz-peaked, and the semi-simultaneous spectra shown in Fig. 1a show that the shape of the spectrum changes depending on the state of the activity. For comparison, the semisimultaneous spectra of a distant $(z=2.624)$ classical GPS source B0742+103 are shown in Fig. 1b. This genuine GPS source is only moderately variable, and the turnover frequency stays more or less the same, regardless of the level of variability. With the first two spectra, both sources could be classified as non-variable GPS sources, but longer monitoring reveals the difference in their behaviour. Thus, Fig. 1 shows that one or two years of monitoring at different frequencies may not be enough to show the long-term changes in the spectrum. Concerning the long-term changes in the radio spectrum, B0238-084 is the only source in this sample that resembles the high-variability sources with spectra becoming inverted during the outbursts that were found to be common among the quasar-type GPS sources identified in the literature (Paper I).

The source B1543+005, with a high number of observations at the Michigan frequencies (Fig. 2), was a borderline case between " $n$ " and "s" classes. We decided to include it in class " $n$ " 
because of its small size $(59 \mathrm{pc})$ as reported by Jeyakumar et al. (2000). Other sources exhibiting steep spectra had either too low a possible turnover frequency (both in the observer's and the rest frame), too high a variability, or no information on size or redshift.

B1843+356 (Fig. 2) has the steepest rise in the spectrum below the turnover, $\alpha_{\text {below }}=+2.25$, which is close to the theoretical maximum for synchrotron radiation. However, no information on the epochs of the observations at frequencies below the turnover is available so it is likely that the spectral index does not describe simultaneous observations. There is variability at the high frequency end of the spectrum $(37-43 \mathrm{GHz})$. The source has been observed a few times at Metsähovi, but only this one flaring point has been detected, while other observations have been non-detections. This, in addition to the low CATS/VLAC data point at $43 \mathrm{GHz}$, implies that there is variability beyond our GPS criteria, so we labelled the spectrum of this source as "convex".

In addition to $\mathrm{B} 1843+356$, there are three sources with apparently clean flat or convex spectra (in Fig. 2) - B0651+410, $\mathrm{B} 1622+665$ and $\mathrm{B} 2323+790$ - that have a recent flux density measurement high above the fitted level. These data points were obtained at $37 \mathrm{GHz}$ at Metsähovi whereas there are several observations of each source at $37 \mathrm{GHz}$ when the source was not detected at all. This implies that the sources spend most of their time at low levels but have flares and thus considerable variability at high frequencies as well. These sources require further monitoring to reveal their variability behaviour. It is highly possible that other "clean" sources will also be caught flaring as the monitoring continues.

The spectrum of some of the flat/steep-spectrum sources (Fig. 6): B0316+162, B0320+053, B1638+124, and B2210+016 seem to rise a little just below the turnover. But since the rise is so weak and could be explained by variability observed at some nearby frequencies, these sources were left out of the convex class. In reality, these or other flat/steep-spectrum sources could turn out to be CSS sources if the feature that now seems to be a flat plateau is actually the top of the turnover. With data missing at the low MHz-frequencies, we cannot at present determine this.

\section{Discussion}

The number of genuine GPS sources in the galaxy-type samples seems to be significantly lower than $100 \%$. In our sample, only $\sim 30 \%$ of all GPS galaxies identified as such in the literature had convex spectra peaking at GHz-frequencies, and the possibility of variability must be considered, at least for the sparsely observed sources. The identification was left uncertain for another $\sim 30 \%$ due to insufficient data. Some of these sources can be genuine GPS sources, whereas some of them will probably prove to be too flat or variable, when observed during an outburst.

For a reliable GPS classification, two things are needed: 1) broadband spectral data in the radio band, preferably at several epochs, to define the turnover, and 2) long-term monitoring data at several frequencies to determine whether the source flux density is variable at any or all frequencies. For only a small fraction of the sources, a sufficient observational record fulfils these requirements. We have done our best with the best data at hand and are aware that the classification of some of these sources will change when more data have been obtained.

For 61 sources, there is no monitoring data available at any frequency band, or the dated observations only span less than two years. Out of these, 11 were GPS sources, 23 " $n$ "-sources and the rest were flat- or steep-spectrum sources. For 16 sources out of all the sources in the sample, we had continuous monitoring data covering more than 10 years, nine of which were genuine GPS sources. In fact, most of the extensively monitored genuine GPS sources had data at several frequencies from well over 20 years. If no variability has been detected in this time span, it is valid to call these sources non-variable during the era of radioastronomical observations. However, the most monitored frequencies were the UMRAO frequencies, 4.8, 8, and 14.5 $\mathrm{GHz}$, so the monitoring does not extend to the inverted part of the spectrum below the turnover frequency. Thus, there is no information about the behaviour of the sources at low frequencies.

The characteristic variability in certain frequency bands seems to be different in different sources, including also the genuine GPS sources. This effect is interesting, and will be studied in more detail in our forthcoming paper (Torniainen et al., in preparation).

To study the effects of long-term sampling in the calculated variability indices, we plotted $\operatorname{Var}_{\Delta S}$ of the data bin with the highest fractional variability and the number of observations $\mathrm{N}_{o b s}$ in that bin for each source (Fig. 3). For the genuine GPS sources, the frequency of bin with the highest variability varied from $0.2 \mathrm{GHz}$ to $37 \mathrm{GHz}$, and the median value was $8.8 \mathrm{GHz}$. For the sources with convex spectra, the greatest $\operatorname{Var}_{\Delta S}$ was in the bins centred at high frequencies $(12-37 \mathrm{GHz})$. For other source classes in our sample, the bin with the greatest variability was typically centred at the frequency of $4.3 \mathrm{GHz}$, because of the extensive UMRAO monitoring at $4.8 \mathrm{GHz}$.

When increasing $N_{\text {obs }}$, the path of the $\operatorname{Var}_{\Delta S}$ vs. $N_{\text {obs }}$ point of a genuine non-variable GPS source should approach a vertical line indicating a low value of $\operatorname{Var}_{\Delta S}$. Indeed, there are genuine GPS sources that maintain their GPS classification, even if there is a large number of observations per bin $\left(N_{\mathrm{obs}} \sim 100\right)$. Unfortunately, this plot does not give any information on the timescales in which the observations have been made, so the result is only suggestive. The lack of date information in the CATS database prevents us from taking the total time span of observations into account.

Another problem is that, in our data taken from, e.g., the CATS database, there are multiple entries for a single measurement. If a data point is quoted in several publications, which are all extracted into our database, the same value is then represented several times. This mainly affects only the low- $N_{\text {obs }}$ end of the plot, because the effect is insignificant for the sources with numerous observations in the bin.

Compared to the GPS quasars studied in Paper I, the galaxytype GPS sources seem to have a genuinely gigahertz-peaked spectrum more often, but there are also incorrectly classified sources. In other words, the galaxy-type samples are contaminated with variable flat spectrum sources, but not as much as the quasar-type samples. Also, the variability of galaxy-type sources seems to be lower: the mean of the maximum variability index of the genuine GPS galaxies is 1.21 whereas the same number was 1.95 for the genuine GPS quasars from Paper I.

This must be taken with caution, however, because a selection effect plays an important role here. The quasar sample was selected mostly among frequently monitored sources, which have originally been selected for monitoring because of their interesting behaviour, so they are prone to exhibiting variability. Many of the galaxies, however, have only a few observations; and with such sparse data sets, it is impossible to confirm any variability, no matter how high it is in reality.

It must also be kept in mind, when interpreting the statistics of our quasar sample, that there were only two genuine GPS 

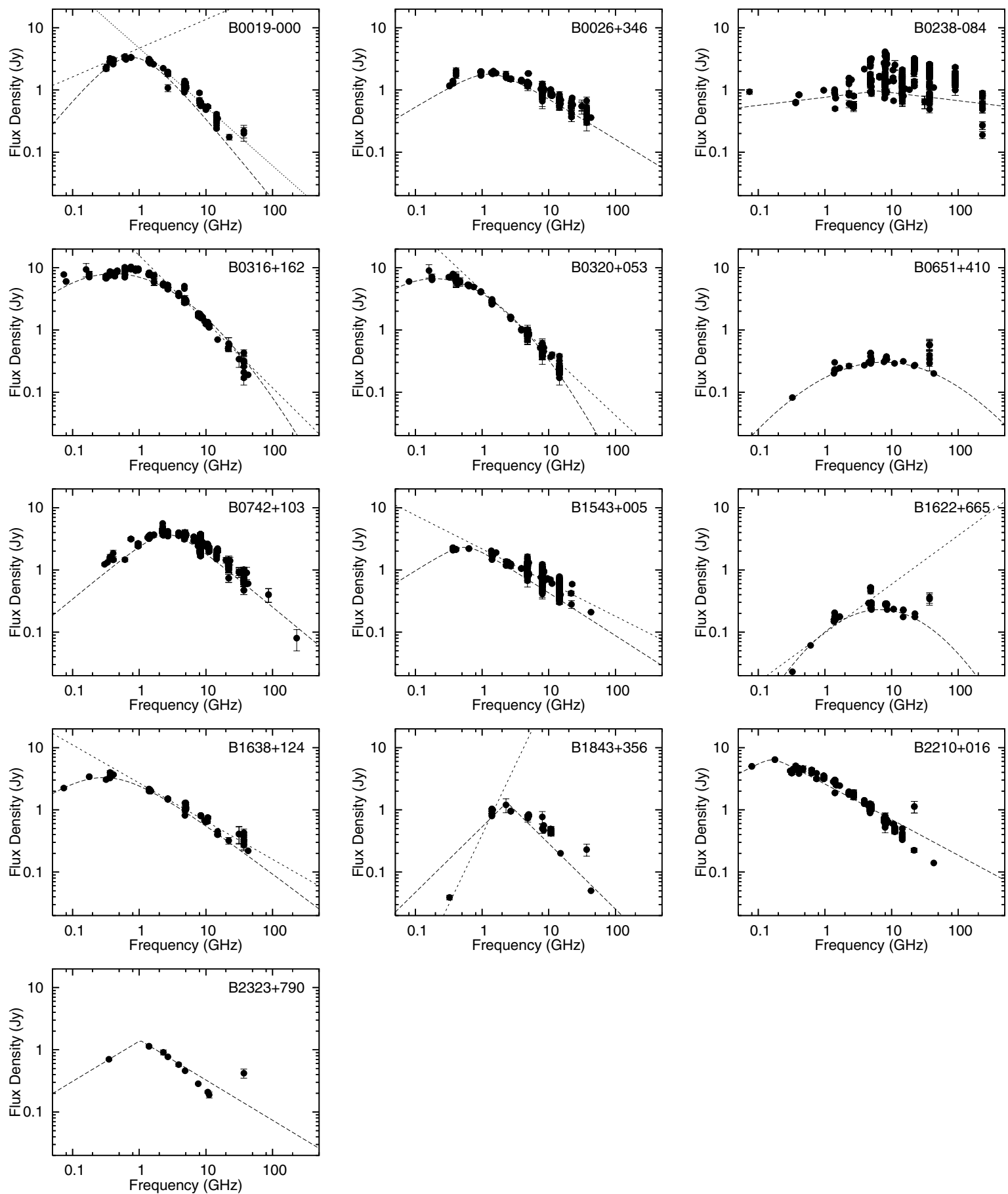

Fig. 2. Radio spectra of galaxies discussed in Sect. 4.1.

quasars and one optically unidentified object, which in this case was assumed to be a quasar, among the 29 previously identified GPS or HFP quasars. We cannot make any firm conclusions about a source population based on the statistics derived from a sample of three sources. More quasar-type GPS sources should be studied to increase the number of the genuine GPS quasars and thus enable more solid statistics on the population. Our forthcoming paper (Torniainen et al., in preparation) will present a statistical study of a large sample of GPS quasars and galaxies identified in the literature.

As in Paper I, the motivation for this study was to determine the effect that the GPS sources will have on ESA's Planck mission $^{3}$ to be launched in 2008 to measure the anisotropies of the cosmic microwave background. These results imply a complicated situation. No reliable prediction, as whether a source is detectable by Planck, can be made based on the classification from the literature. Many of the sources classified as GPS sources in the literature, and thus thought to be non-variable, can exhibit variability and appear unforeseeably bright at high radio frequencies during the Planck observations. Examples are the four sources mentioned at the end of Sect. 4.1, which were detected at Metsähovi even though their historical data led us to expect non-detections.

\footnotetext{
${ }^{3}$ http://www.rssd.esa.int/Planck
} 


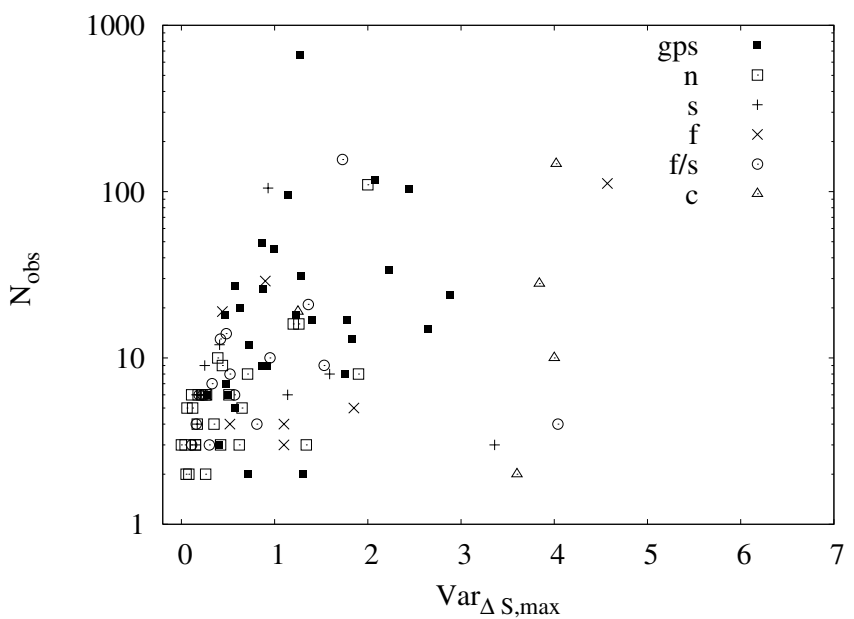

Fig. 3. Fractional variability index for the bin with the highest value and number of observations in the same bin.

\section{Conclusions}

We have gathered a large database of radio observations on 96 galaxy-type GPS sources. After plotting their radio spectra and calculating the spectral and variability indices we found that only about $30 \%$ of them clearly had a gigahertz-peaked spectrum, and the peaked spectrum was possible for another $\sim 30 \%$ but could not be confirmed due to lack of data. The rest of the sources were flat-spectrum sources, some of them showing significant variability, or steep-spectrum sources that could turn out to be CSS sources if the low-frequency end of the spectrum proved to be inverted.

These results are in accordance with the results from our earlier work. In Paper I we studied a sample of mostly quasar-type GPS sources classified in the literature and discovered a similar result: only a small fraction of the quasar-type GPS sources were genuine non-variable convex-spectrum sources. It would seem that classification of the galaxy-type GPS sources is on somewhat firmer ground than classification of the GPS quasars. The quasar sample was smaller than the galaxy sample, with only three genuine GPS quasars. Also, the possible selection effect must be taken into account. The quasar sample was selected among sources from the Metsähovi monitoring project, whereas the galaxy sample was a complete sample from the bright GPS samples presented in the literature.

Nevertheless, the results of the GPS studies presented in the literature, regardless of whether discussing the galaxy-type or quasar-type GPS sources, must be taken with caution, since the original samples are very likely to be highly contaminated.
Monitoring of the GPS sources continues at Metsähovi. In addition, we are observing the sources with the RATAN-600 telescope in order to obtain simultaneous spectra. The monitoring will be continued as long as needed to gain information on the long-term variability behaviour of these sources at high radio frequencies.

Acknowledgements. The authors have made use of the database CATS (Verkhodanov et al. 1997) of the Special Astrophysical Observatory. The authors acknowledge the support of the Academy of Finland to the Metsähovi observing projects. UMRAO is supported in part by funds from the NSF and by funds from the University of Michigan Department of Astronomy. This research made use of the NASA/IPAC Extragalactic Database (NED), which is operated by the Jet Propulsion Laboratory, California Institute of Technology, under contract with the National Aeronautics and Space Administration. I.T. wishes to thank T. Hovatta and E. Nieppola for fruitful conversations. We also thank the anonymous referee for very detailed and constructive comments.

\section{References}

Aller, H. D., Aller, M. F., Latimer, G. E., \& Hodge, P. E. 1985, ApJS, 59, 513 Baum, S. A., O’Dea, C. P., de Bruyn, A. G., \& Murphy, D. W. 1990, A\&A, 232, 19

Bolton, R. C., Chandler, C. J., Cotter, G., et al. 2006, MNRAS, 370, 1556 Dallacasa, D., Stanghellini, C., Centonza, M., \& Fanti, R. 2000, A\&A, 363, 887 de Vries, W. H., Barthel, P. D., \& O'Dea, C. P. 1997, A\&A, 321, 105 Fanti, C., Pozzi, F., Fanti, R., et al. 2000, A\&A, 358, 499

Fanti, C., Fanti, R., Dallacasa, D., et al. 1995, A\&A, 302, 317

Gopal-Krishna \& Wiita, P. J. 1991, ApJ, 373, 325

Gugliucci, N. E., Taylor, G. B., Peck, A. B., \& Giroletti, M. 2005, ApJ, 622, 136

Jeyakumar, S., Saikia, D. J., Pramesh Rao, A., \& Balasubramanian, V. 2000, A\&A, 362, 27

Kovalev, Y. A., Kovalev, Y. Y., \& Nizhelsky, N. A. 2000, PASJ, 52, 1027

Mantovani, F., Junor, W., Fanti, R., Padrielli, L., \& Saikia, D. J. 1994, A\&A, 292, 59

Marecki, A., Barthel, P. D., Polatidis, A., \& Owsianik, I. 2003, PASA, 20, 16

Murgia, M., Fanti, C., Fanti, R., et al. 1999, A\&A, 345, 769

O’Dea, C. P. 1998, PASP, 110, 439

O'Dea, C. P., Stanghellini, C., Baum, S. A., \& Charlot, S. 1996, ApJ, 470, 806

O'Dea, C. P., Gallimore, J., Stanghellini, C., Baum, S. A., \& Jackson, J. M. 2005, AJ, 129,610

Orienti, M., Dallacasa, D., Tinti, S., \& Stanghellini, C. 2006, A\&A, 450, 959 Phillips, R. B., \& Mutel, R. L. 1982, A\&A, 106, 21

Snellen, I. A. G., Schilizzi, R. T., de Bruyn, A. G., et al. 1998, A\&AS, 131, 435

Snellen, I. A. G., Schilizzi, R. T., Bremer, M. N., et al. 1999, MNRAS, 307, 149

Stanghellini, C., Baum, S. A., O’Dea, C. P., \& Morris, G. B. 1990, A\&A, 233, 379

Stanghellini, C., Dallacasa, D., O’Dea, C. P., et al. 2001, A\&A, 379, 870

Stanghellini, C., O'Dea, C. P., Dallacasa, D., et al. 2005, A\&A, 443, 891

Teräsranta, H., Tornikoski, M., Mujunen, A., et al. 1998, A\&A, 132, 305

Tinti, S., Dallacasa, D., de Zotti, G., Celotti, A., \& Stanghellini, C. 2005, A\&A, 432,31

Torniainen, I., Tornikoski, M., Teräsranta, H., Aller, M. F., \& Aller, H. D. 2005, A\&A, 435, 839 (Paper I)

Tornikoski, M., Jussila, I., Johansson, P., Lainela, M., \& Valtaoja, E. 2001, AJ, 121,1306

Verkhodanov, O. V., Trushkin, S. A., Andernach, H., \& N., C. V. 1997, ed. Gareth Hunt, \& H. E. Payne, ASP Conf. Ser., 125, 322 
I. Torniainen et al.: Radio continuum spectra of gigahertz-peakedspectrum galaxies, Online Material p 1

\section{Online Material}


I. Torniainen et al.: Radio continuum spectra of gigahertz-peakedspectrum galaxies, Online Material p 2
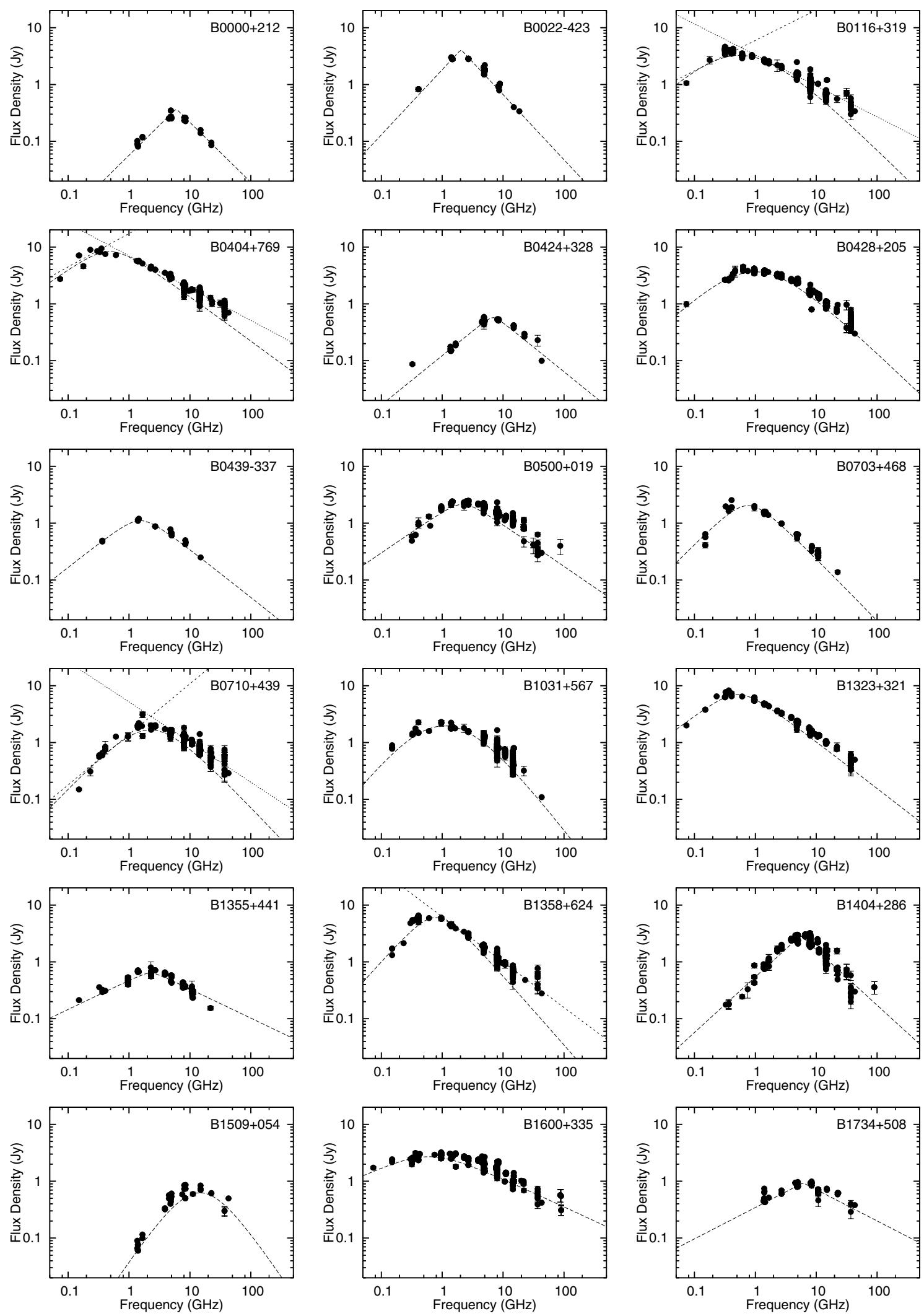

Fig. 4. Radio spectra of sources with GPS spectra. 
I. Torniainen et al.: Radio continuum spectra of gigahertz-peakedspectrum galaxies, Online Material p 3
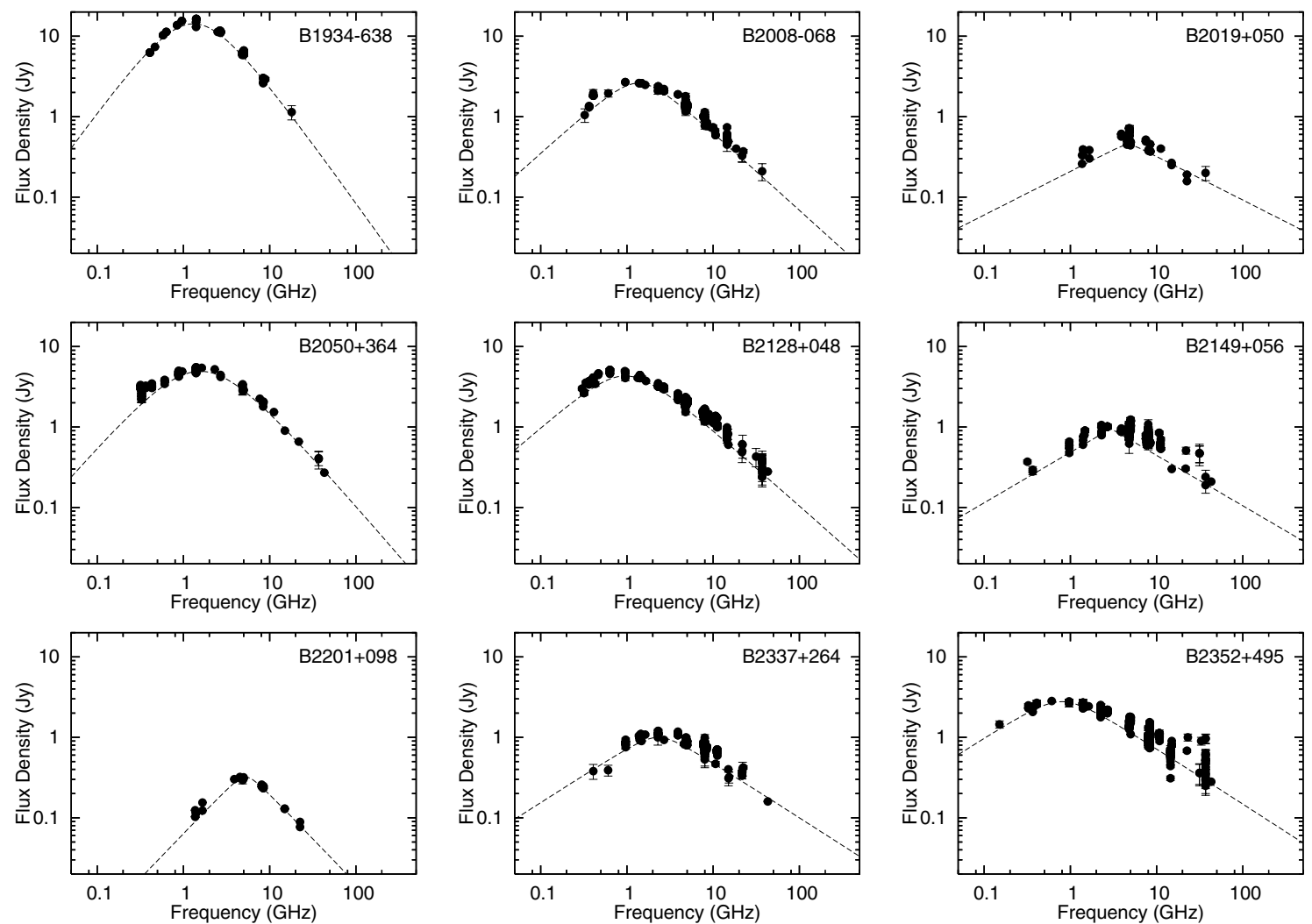

Fig. 4. Continued. 
I. Torniainen et al.: Radio continuum spectra of gigahertz-peakedspectrum galaxies, Online Material p 4
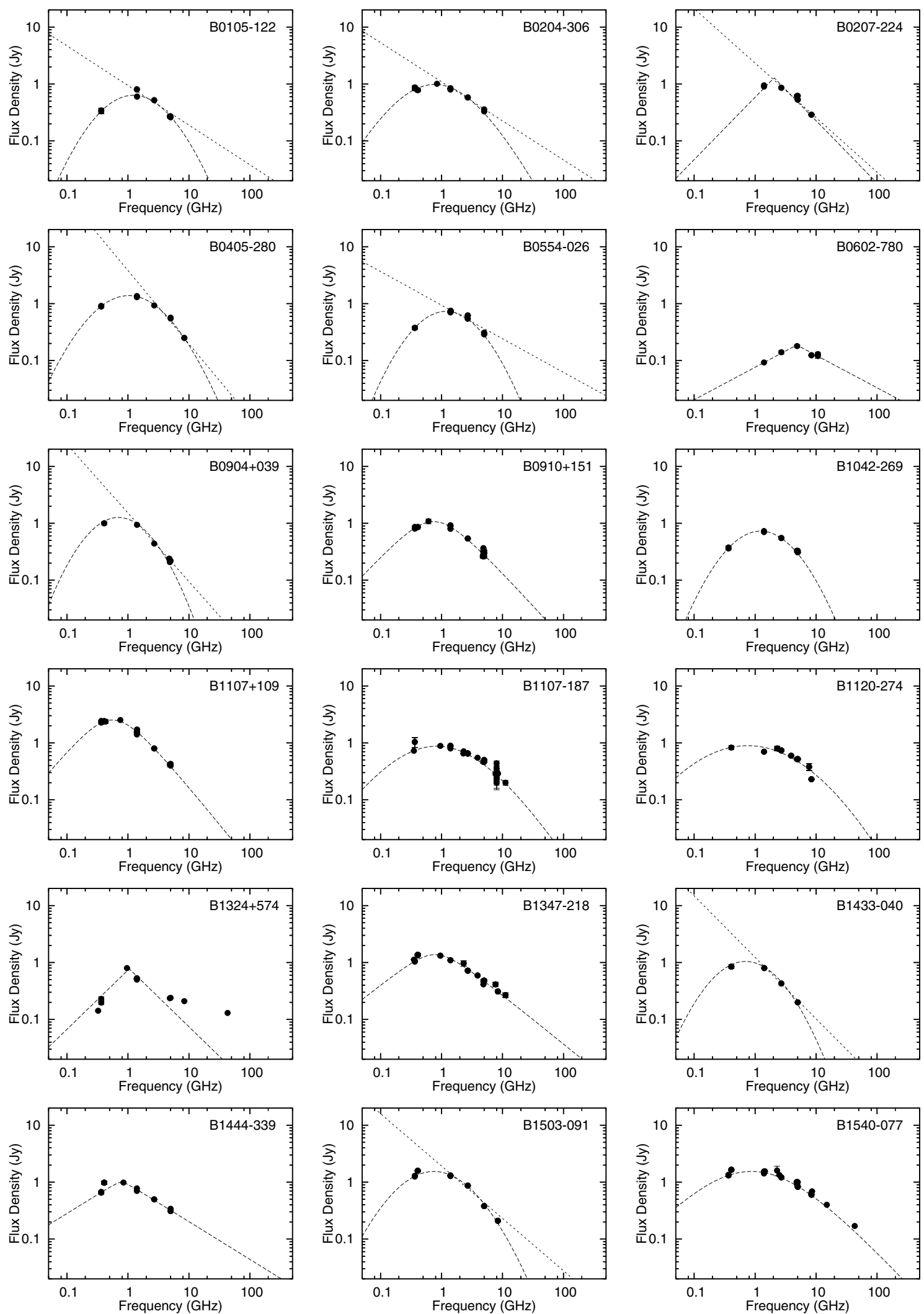

Fig. 5. Radio spectra of sources with insufficient data for GPS classification. 
I. Torniainen et al.: Radio continuum spectra of gigahertz-peakedspectrum galaxies, Online Material p 5
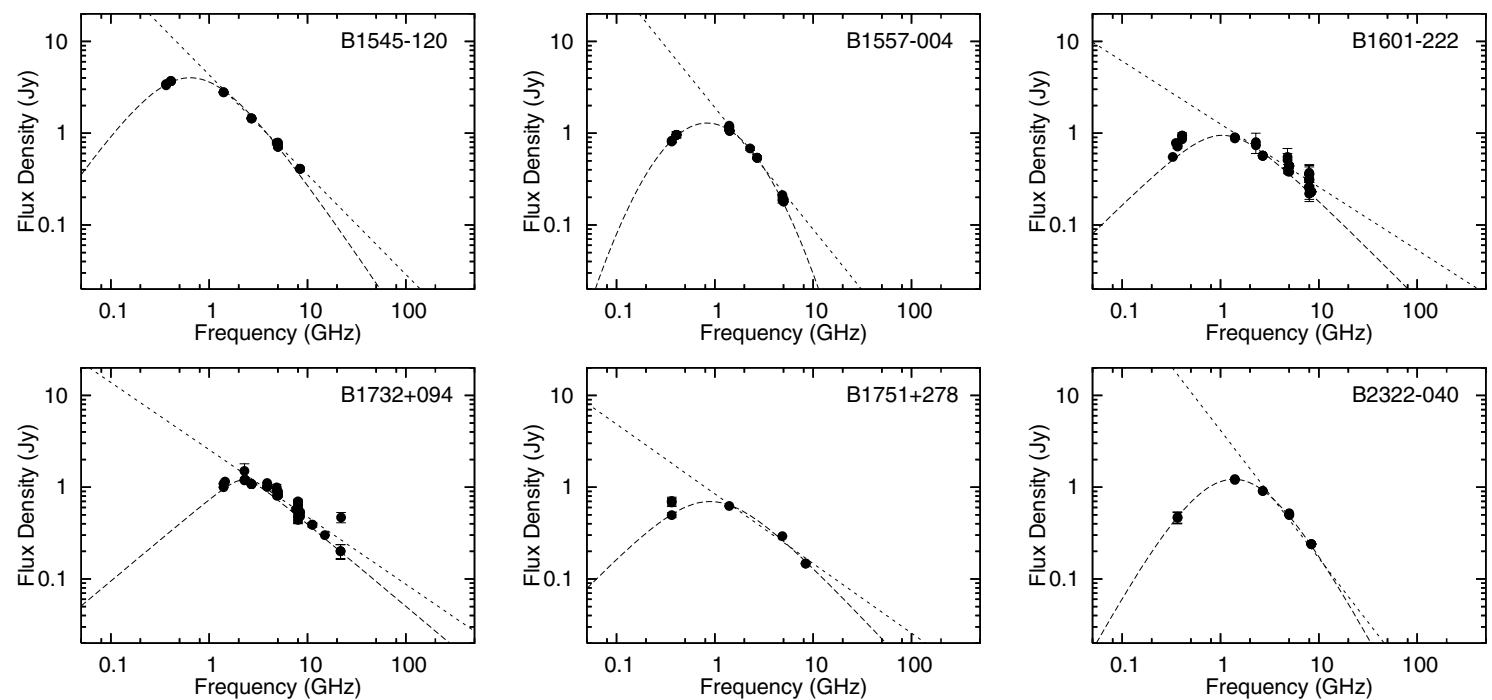

Fig. 5. Continued.
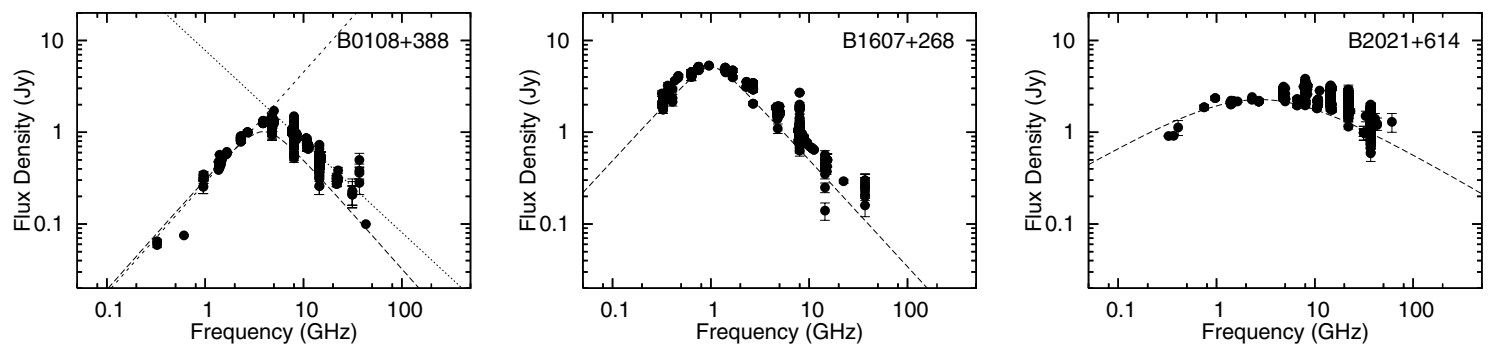

Fig. 6. Radio spectra of sources with convex spectra. 
I. Torniainen et al.: Radio continuum spectra of gigahertz-peakedspectrum galaxies, Online Material p 6
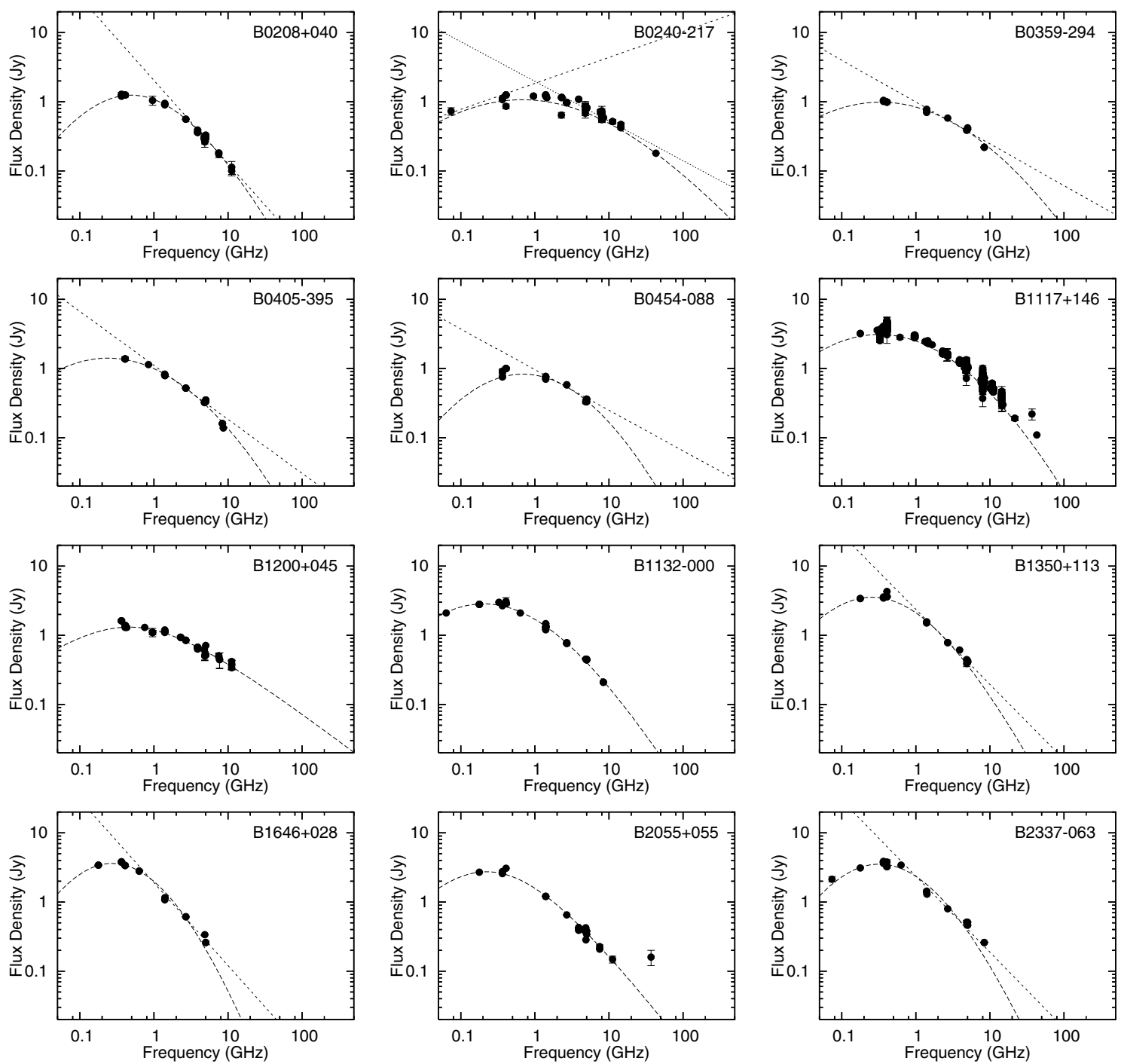

Fig. 7. Radio spectra of sources with flat/steep spectra.
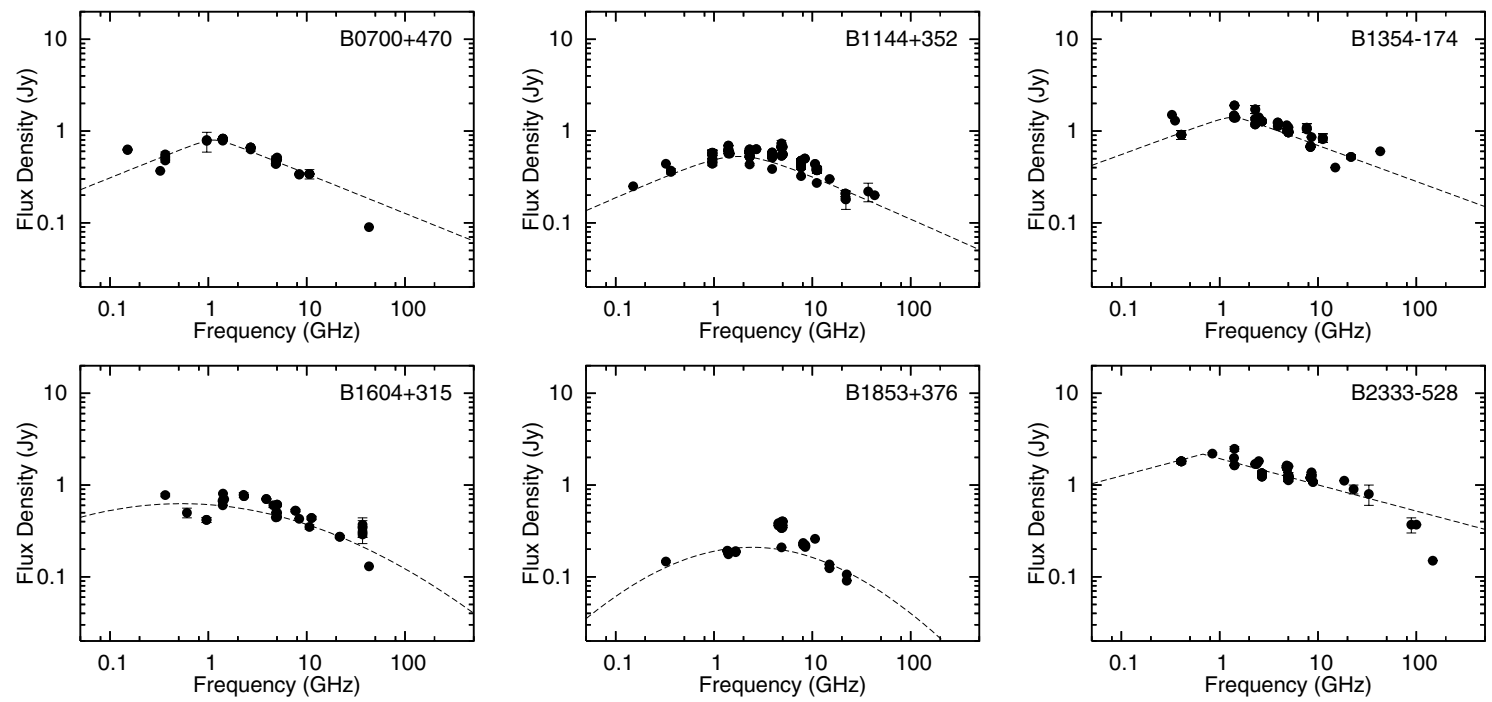

Fig. 8. Radio spectra of sources with flat spectra. 
I. Torniainen et al.: Radio continuum spectra of gigahertz-peakedspectrum galaxies, Online Material p 7
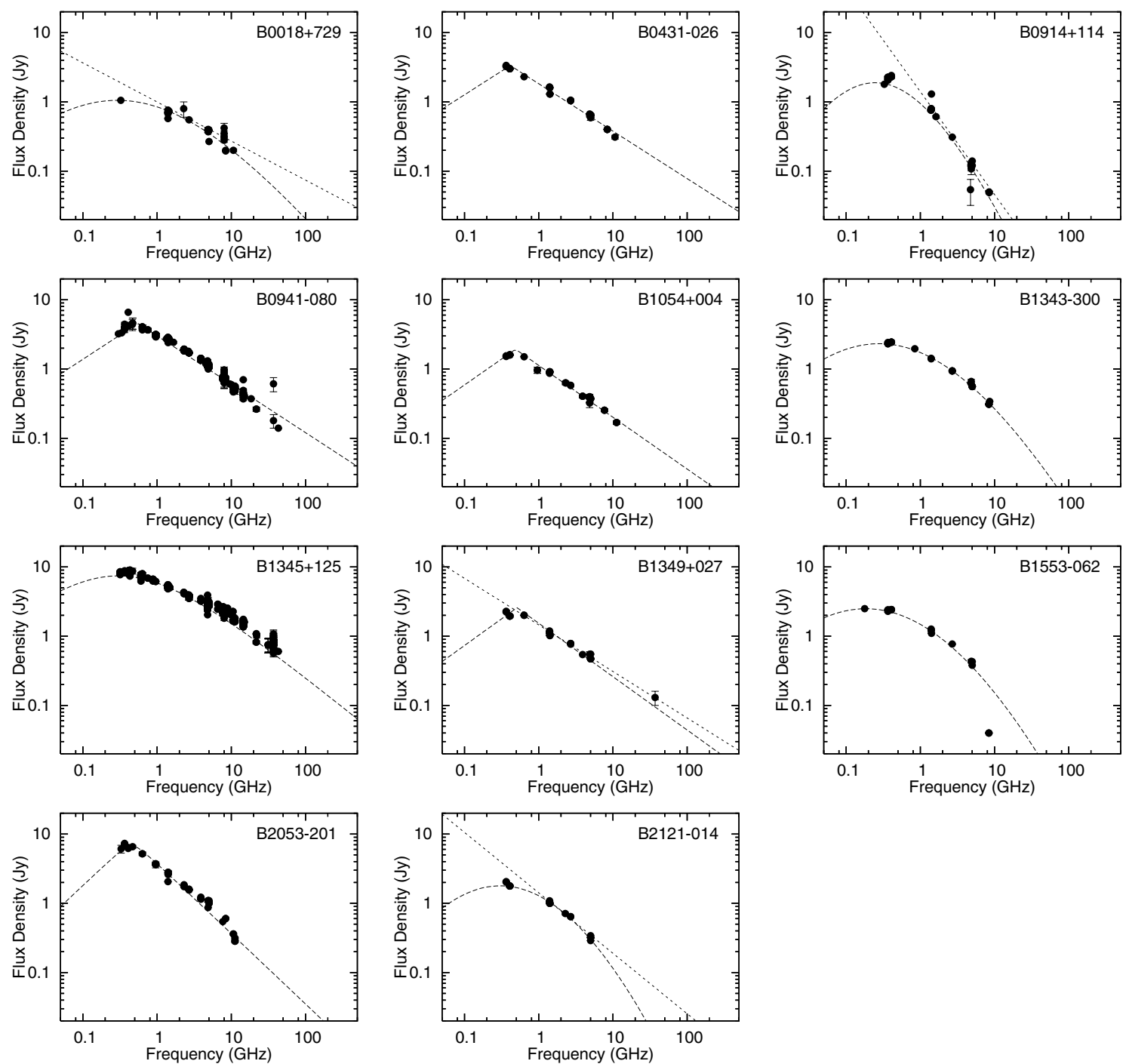

Fig. 9. Radio spectra of sources with steep spectra. 